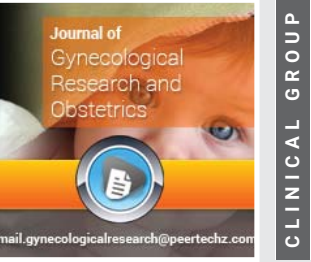

\section{Preventive oral health care}

\section{for mitigating the COVID-19}

\section{induced adverse maternal}

\section{outcomes: View point from Sri Lanka on opportunities in crisis}

\author{
Irosha Perera1*, Manosha Perera², Nadisha Ratnasekera', \\ Chandana Gajanayake ${ }^{3}$ and Migara $\mathrm{Epa}^{4}$ \\ ${ }^{1}$ Preventive Oral Health Unit, National Dental Hospital (Teaching) Sri Lanka, Ward Place, Colombo \\ 700700, Sri Lanka \\ ${ }^{2}$ Alumnus, School of Dentistry and Oral Health, Griffith University, Gold Coast, Qld, Australia \\ ${ }^{3}$ Office of Deputy Director, National Hospital Sri Lanka, Colombo, Sri Lanka \\ ${ }^{4}$ Office of Deputy Director, National Dental Hospital (Teaching) Sri Lanka, Ward Place, Colombo 7, Sri \\ Lanka
}

Received: 30 September, 2021

Accepted: 13 October, 2021

Published: 14 October, 2021

*Corresponding author: Dr. Irosha Perera, Preventive Oral Health Unit, National Dental Hospital (Teaching) Sri Lanka, Ward Place, Colombo 700700, Sri Lanka, Tel: +94 11 2693106, +94 112678834; Fax: +94 112678753 E-mail: irosha_rukmali@yahoo.com

ORCID: https://orcid.org/0000-0002-8250-0169

Copyright: @ 2021 Perera l, et al. This is an openaccess article distributed under the terms of the Creative Commons Attribution License, which permits unrestricted use, distribution, and reproduction in any medium, provided the original author and source are credited.

https://www.peertechzpublications.com

\section{Check for updates}

\title{
Abstract
}

Pregnancy marks a unique milestone in woman's life cycle enriched with an array of physical, psychological, social and even spiritual needs. In this context, an optimal level of oral health status has become fundamental to overall health and well-being of a pregnant woman. This is evident more than ever before, for preventing exacerbation of poor oral health in pregnancy, possible control of adverse pregnancy outcomes whilst ensuring low risk for early childhood dental caries for the offspring. Nevertheless, pregnancy renders special oral health needs for a woman compounded by changes in dietary habits, oral hygiene practices and hormonal changes thereby increasing the risk of dental caries and periodontitis. The possible link between Periodontitis and adverse outcomes in pregnancy is well known. The pandemic caused by acute respiratory syndrome coronavirus 2 (SARS-CoV-2) compounded by emergence more virulent strains not only indelibly impacted on population, countries, economies and health systems across the globe but exposed vulnerable population groups to pervasive health catastrophes. There is accumulating concerns on periodontitis linking with severe complications of Covid-19 attributed to commonalities of exacerbated inflammatory responses. Moreover, adverse Covid-19 induced maternal and fetal outcomes has garnered attention of researchers based on the previous knowledge indicating adverse outcomes attributed to epidemics of human corona viruses. However, it is not known how maternal oral hygiene status and burden of periodontitis impact on Covid-19 induced adverse outcomes in pregnancy. However, it could be rational to argue that poor oral health of a pregnant woman could elevate her risks for adverse health outcomes. Oral health care in pregnancy has received recognition as an essential element of comprehensive antenatal health care provision. However, the persistent global public health emergency of Covid-19 has profoundly impacted on availability of and accessibility to routine oral health care services. Therefore, accessing routine oral health care services poses serious causes for concern among pregnant women. Against this backdrop, present opinion attempts to explore and expound the potential of preventive oral health care could offer to mitigate the potential risks of Covid-19 induced adverse maternal outcomes embracing the context of Sri Lanka: a developing lower middle-income country.

\section{Introduction}

Pregnancy denotes a unique physiological milestone in a life-cycle of a woman enriched with an array of physical, psychological, social and even spiritual needs. Nevertheless, health outcomes of pregnant women are increasingly being challenged in the era of persistent Covid-19 public health emergency negatively impacting on health care systems, economies and population across the globe [1]. There is accumulating evidence on increased severity of Covid-19 infection on unvaccinated pregnant women especially in late trimesters, having non-communicable diseases spanning from 
vertical transmission to maternal, fetal and neonatal mortality [2-5]. Moreover, it is well-known that pregnancy poses increased oral health care needs to a woman and increased risk for oral diseases such as dental caries and periodontal diseases predominantly attributed to altered dietary habits and hormonal changes compounded by less optimal oral hygiene practices and issues in accessing oral health care services [6-8]. Research evidence suggests periodontitis; the polymicrobial chronic inflammatory disease of the periodontium (tooth supporting structures) could be associated with the elevated risk for gestational diabetes mellitus and adverse pregnancy outcomes thus warranting further research with methodological rigour [9-13]. Furthermore, the resemblance of placental microbiome with the maternal oral microbiome has gained attention of researchers due to its practical implications linking maternal periodontitis with adverse pregnancy outcomes mediated by prominent periodontopathic bacteria such as Porphyromonas gingivalis and Fusobacterium [13-16]. Therefore, oral health care for pregnant women garnered acceptance as an essential element in antenatal care, with increased focus on preventive oral health care [17]. Besides, there is accumulating evidence linking periodontitis with increased severity of Covid-19 and adverse health outcomes mediated by existing noncommunicable diseases especially among older adults [18-20]. Nevertheless, it is not known how maternal periodontal disease may impact on adverse maternal, fetal and neonatal outcomes within the context of Covid-19 pandemic among pregnant women. This impact could be necessarily confounded by receiving the protective vaccination. Against this backdrop, the goal of this opinion piece attempts to explore and expound the potential of preventive oral health care could offer to mitigate the potential risks of Covid-19 induced adverse maternal outcomes embracing the context of Sri Lanka: a developing lower middle-income country.

\section{Periodontitis, systematic health and COVID-19}

There is accumulating evidence to speculate the inextricable relationship of oral health with systemic health and vice versa [20-21]. Specifically, periodontal disease has been linked with diabetes, metabolic syndrome, obesity, eating disorders, liver disease, cardiovascular disease, hypertension, Alzheimer disease, rheumatoid arthritis and ageing [20,21]. Despite recognition of bidirectional relationships, the potential for multiple comorbidities, relationships, and connections (multimodal relationships) has been explored [22]. Periodontal disease (PD) is known to comprise a group of diseases containing inflammatory aspects of the host and dysbiotic events that affect periodontal tissues with systemic implications [2325]. Further, periodontitis has identified as a risk factor for pneumonia and the exacerbation of chronic obstructive pulmonary disease, presumably attributed to the aspiration of saliva contaminated with periodontopathic bacteria into the lower respiratory tract [26]. Thus, there is a biologically plausible explanation for elevated risk for aggravation of Covid-19 and subsequent mortality. Periodontopathic bacteria have been isolated from the bronchoalveolar lavage fluid of patients with COVID-19, thereby speculating the possibility of periodontitis as a risk factor for COVID-19 aggravation. This could be attributed to spectacular involvement of inflammatory mediators and dysbiotic events of Covid-19 infection itself and systemic effects of periodontitis [23,24]. Therefore, there is evidence to suggest that Covid-19 complications are caused by severe inflammatory reaction such as cytokine flux thus sharing some common signals with periodontal disease [18-19, 23-24]. Supporting those notions, as reported by a recent casecontrol study conducted using the national electronic health records of the State of Qatar there was a significant association between periodontitis and adverse Covid-19 complications. Cases included patients who suffered COVID-19 complications (death, ICU admissions or assisted ventilation), and controls comprised COVID-19 patients discharged without major complications [18]. Periodontal conditions have been assessed by dental radiographs from the given database arriving at the definition of periodontitis when bone loss was detected at two or more non-adjacent teeth after accounted for local factors [18]. Subsequently, researchers categorized periodontal disease as "periodontally healthy or initial periodontitis (Stages 0-1)": bone loss less than the coronal third of the root length (15\%) in OPGs, or $\leq 2 \mathrm{~mm}$ in bitewing radiographs and "Periodontitis (Stages 2-4)": bone loss more than the coronal third of the root length ( $>15 \%$ ) in OPGs, or $>2 \mathrm{~mm}$ in bitewing radiographs [18]. As emerged from the findings of this study adjusted for potential confounders, periodontitis was associated with COVID-19 complications including death $(\mathrm{OR}=8.81,95 \% \mathrm{CI}$ 1.00-77.7), ICU admission ( $\mathrm{OR}=3.54,95 \%$ CI 1.39-9.05) and need for assisted ventilation ( $\mathrm{OR}=4.57,95 \% \mathrm{CI} 1.19-17.4$ ) [18]. Study findings further revealed that blood levels of white blood cells, D-dimer and C Reactive Protein were significantly higher in COVID-19 patients having periodontitis. The findings therefore, supported the association of periodontitis with adverse COVD-19 outcomes and elevated blood levels of inflammatory biomarkers of those worst outcomes [18]

Another case-control study conducted in this regard included patients who had positive real-time reverse transcription polymerase chain reaction (rt-PCR) results for SARS-CoV-2 infection as cases, and patients with negative results as controls [19]. The periodontal examination involved recording the plaque scores, calculus scores, tooth mobility, gingival bleeding, probing depth, recession, and clinical attachment level (CAL) [19]. As revealed by the findings, significant associations were detected among mean plaque scores $\geq 1$ (odds ratio (OR), 7.01; 95\% CI: 1.83 -26.94), gingivitis (OR, 17.65; 95\% CI: $5.95-52.37$ ), mean CAL $\geq 2 \mathrm{~mm}$ (OR, 8.46; 95\% CI: 3.47 - 20.63), and severe periodontitis (OR, 11.75; 95\% CI: 3.89 - 35.49) with COVID-19 [19]. As the study included small sample size there are wider confidence intervals. Nevertheless, the findings suggest plausible link of poor oral hygiene with Covid-19. As indicated by their findings, authors highlighted the importance of maintaining periodontal health and good oral hygiene for prevention and management of Covid-19 [19].

Further, substantiating this notion a recent study unraveled the molecular links between periodontitis and COVID-19 [26]. Findings of this study revealed that the culture supernatant of the periodontopathic bacterium Fusobacterium nucleatum 
(CSF) upregulated the SARS-CoV-2 receptor angiotensinconverting enzyme 2 in A549 alveolar epithelial cells and CSF induced interleukin (IL)- 6 and IL- 8 production by both A549 and primary alveolar epithelial cells [26]. In nutshell, those findings suggested that when patients with mild COVID-19 frequently aspirate periodontopathic bacteria, SARS-CoV-2 infection could be promoted, thus aggravating inflammation of lower respiratory tract in the presence of viral pneumonia [26]. Therefore, it is prudent to speculate, from public health perspectives through to molecular perspectives; there is compelling evidence to suggest optimal oral health could be an important protective factor to mitigate the adverse outcomes of Covid-19 infection. Preventive oral health care is the way forward to maintain optimal oral health status by empowering individuals and risk groups to gain a better control over their own oral health status.

Availability of and accessibility to preventive oral health care services become crucial for providing oral health care for pregnant women. Delivering oral health advice over the phone or via other telecommunication platforms to pregnant women within the context of health service restriction makes a promising approach in this persistent Covid-19 crisis. Sri Lanka as a lower-middle-income developing country possessing a vibrant public health care delivery model to cater to all citizens in the country incorporated oral health care to antenatal health care package way back in 2009 [6]. Preventive oral health care became an integral component of this service package [26-28]. Nevertheless, pregnant women from socially disadvantaged and minority background encounter a plethora barriers in accessing oral health care services comprising cost of services, dental anxiety/fear and discrimination [27]. The unique public health delivery model of Sri Lanka has garnered recognition for its effectiveness in improving maternal and child health outcomes [29].

\section{Oral health programme for pregnant women in Sri Lanka}

Sri Lanka possess an estimated mid-year population of 21.9 million for the year 2020 [30] catered by the public health care delivery model. There is a well-organized maternal and child health programme in the country making antenatal care a key-element of it [6]. Pregnant women are eligible to receive antenatal care free of charge in the public health system, irrespective of their socioeconomic status and geographic location, and have a minimum of five contacts with the primary health team during pregnancy. This service model accomplished 98\% coverage [6]. Oral health care was incorporated into this service model supported by the evidence of high burden of oral diseases among both urban and rural pregnant women in Sri Lanka which was more skewed towards the rural women [6]. As emerged from the findings of a study, the mean Decayed Missing and Filled Teeth (DMFT) among rural antenatal women were $5.4 \pm 3$, with $2.27( \pm 2.31)$ decayed teeth, $1.25( \pm 1.97)$ missing teeth, and $1.90( \pm 1.89)$ filled teeth. Among urban antenatal women, the mean DMFT was 3.69 ( \pm 3.62) with 1.04 ( \pm 2.15) decayed teeth, 1.07 ( \pm 1.59) missing teeth and $1.59( \pm 2.06)$ filled teeth [6]. Rural antenatal women had a significantly higher experience of decayed teeth $(\mathrm{p}=0.001)$ and filled teeth $(\mathrm{p}=0.026)$, and twice as many untreated dental caries, compared with urban women [6]. Moreover, almost $60 \%$ of rural women presented with bleeding gums. Similarly, the prevalence of calculus was $30.3 \%$ for rural women and $13.5 \%$ for urban women. Against this backdrop, the overall objective of national oral health programme for pregnant women is to promote the oral health status of mothers and their children by providing comprehensive oral health care during the prenatal, antenatal and postnatal periods [31]. Reducing the incidence of complications of poor oral health during pregnancy and adverse pregnancy outcomes are among the specific objectives of this programme [31]. According to the National Oral Health Programme for pregnant mothers, all pregnant mothers need to get screened for oral diseases and receive dental treatment [31]. Therefore, all pregnant women who visit antenatal clinics are being referred to nearby dental clinics such as hospital dental clinics, community dental clinics, $\mathrm{MOH}$ dental clinics etc. Therefore, it is timely to explore how oral health care underpinned by preventive oral health care could mitigate the adverse maternal outcomes induced by Covid-19 infections.

\section{Burden of COVID-19 and maternal health outcomes}

The global pandemic of Covid-19 has indelibly impacted on health systems, countries and economies across the globe. Emergence of new strains of SARS-Cov-2 such as delta variant marked an exponential infectivity, high morbidity and mortality across the globe. Adverse maternal health outcomes of Covid-19 underpinned by high risk pregnant women characterized by 235-years, obesity, having non-communicable diseases were reported from many countries. Accordingly, as reported by a recent systematic review based on avilable evidence, Covid-19 related maternal mortality mostly occurred among women having previous co-morbidities whilst neo-natal mortality was predominantly attributed to prematurity rather than infection [5]. Nevertheless, authors warranted further research to determine the magnitude of Covid-19 attributed maternal and perinatal mortality [5]. However, another systematic review that assessed maternal and perinatal outcomes with 108 pregnancies during the early stage of Covid-19 global pandemic reported that the majority of pregnant women presented in third trimester with fever, coughing, lymphocytopenia and the overwhelming majority $(70 \%)$ with elevated C-reactive protein [4]. Furthermore, according to authors despite the majority of mothers delivered their babies with $\mathrm{C}$-sections and discharged without major complications, severe maternal morbidity and attributed to Covid-19 and perinatal deaths were reported indicating the potential vertical transmission of the infection [4]. Therefore, it could be well argued that the maternal, fetal and neonatal outcomes due to Covid-19 have been evolving across the trajectory of Covid-19 global pandemic confounded by emergence of more virulent and transmissible strains. Further research with rigorous methodologies much warranted in this regard from different geographic, socio-cultural and health system contexts of the globe for more conclusive evidence.

Similar to many countries around the globe, Sri Lanka is currently grappling with the third wave of Covid-19 following 
successful combat of first and second waves. Accordingly, adverse maternal outcomes underpinned by 55 maternal deaths attributed to Covid-19 infection were reported with a total 7000 pregnant women getting infected. At the time of writing this view point as per $27^{\text {th }}$ September 2021, a total number of 12,731 Covid-19 related deaths were reported from Sri Lanka with a cumulative total of 514,592 cases (https:// www.worldometers.info/coronavirus/country/sri-lanka/). As at present the Sri Lankan mutation of Covid-19 delta variant is categorized as B.1.617.2.28 with the identification of AY 28 sub-strain. However, the country is currently engaged in a pro-active, vigorous high risk, targeted and population- based vaccination campaign against Covid-19 with the reported complete coverage of $53 \%$. Importantly, as at present $80 \%$ of pregnant women have been vaccinated indicating a positive outcome.

\section{Preventive oral health care model for pregnant wo- men before and within Covid-19 pandemic: Sri Lankan context}

Provision of preventive oral health care comprising oral health awareness for maintaining optimal oral health for the mother and the baby, dietary counseling, tooth brushing advice and demonstrations, provision of oral health awareness leaflets combined with curative oral health care is well evident in the public oral health care delivery model in Sri Lanka [28]. Interactive oral health education sessions are also delivered to pregnant women combined with other health education programmes such as lactation management, nutrition and other maternal \& child health-related topics using antenatal clinics as the health promotion setting [28]. The Preventive Oral Health Unit (POHU) of National Dental Hospital (Teaching) Sri Lanka, the premier, multispecialty, tertiary care public dental hospital conducts a geographically targeted preventive oral health delivery model for socially disadvantaged and culturally diverse urban pregnant women since the year 2011 [28]. Oral health sessions were conducted at antenatal clinics of maternal and child health services Colombo Municipal Council (CMC) region. Oral health awareness, screening for oral diseases and referral for oral health care were essential elements in those sessions conducted by dental surgeons attached to POHU. Performance of this model pertaining to oral health awareness/ screening coverage and referral oral health care for the years 2015-2018 is published elsewhere [28]. Some of the pregnant women from those sessions and other referrals were provided with comprehensive preventive oral health care by POHU. This service model becomes commendable in the light of evidence of low utilization of oral health care services by pregnant women belonging to socially disadvantaged and minority ethnic backgrounds in other countries [27].

Nevertheless, since the detection of the first Sri Lankan Covid-19 patient on $11^{\text {th }}$ March 2020, a stringently imposed island wide lock down was imposed which successfully combatted the first wave of Covid-19 in Sri Lanka [32] Nevertheless, corroborating the scenario in many developed and developing countries across the globe, Sri Lanka has been battling with the second and Delta variant dominant third wave of Covid-19. Therefore, there was persistent breakdown in routine oral health care services delivered by the National Dental Hospital limiting to urgent and essential services $[33,34]$. However, child friendly preventive oral health care services were continued thus facilitating social distancing strategies amidst many challenges [35]. The supportive staff of the hospital was deployed for providing Covid-19 patient care services in other hospitals and treatment centres and it has been the practice to this date. Consequently, such a scenario compounded by physical and social distancing strategies negatively impacted on conducting community oral health sessions for pregnant women at CMC antenatal clinics and providing preventive health care to those women. Therefore, after a lapse, the community oral health sessions were recommenced on the $8^{\text {th }}$ January 2021 , at one antenatal clinic with limited numbers of pregnant women whilst adhering to stringent Covid-19 infection control measures.

Comparison of attendance of pregnant women for preventive oral health care for the year 2019 depicting preCovid-19 era, and for the years of 2020 and 2021 (up to August) presenting Covid-19 era is presented in Figure 1. The findings clearly demonstrate the pervasive negative impact of Covid-19 intermingled with periods of some improvements in accessing preventive oral health care by socially disadvantaged, culturally diverse pregnant women in Sri Lankan context. Nevertheless, the highest access of preventive oral health care by pregnant women over the given periods was reported for July 2020, which continued to some extent to the month of August 2020 thus coinciding with successful control of Covid-19 first wave in Sri Lanka with resumption of routine oral health care services. Nevertheless, the attendance patterns evidently demonstrate the negative impact of emergence of second wave of Covid-19 in late September to October 2020 and devastating third wave in April 2021 for accessing preventive oral health care by pregnant women.

It is not known how oral health status and periodontal condition impacted on Covid-19 induced maternal mortality and morbidity across the globe including Sri Lanka. Nevertheless, as

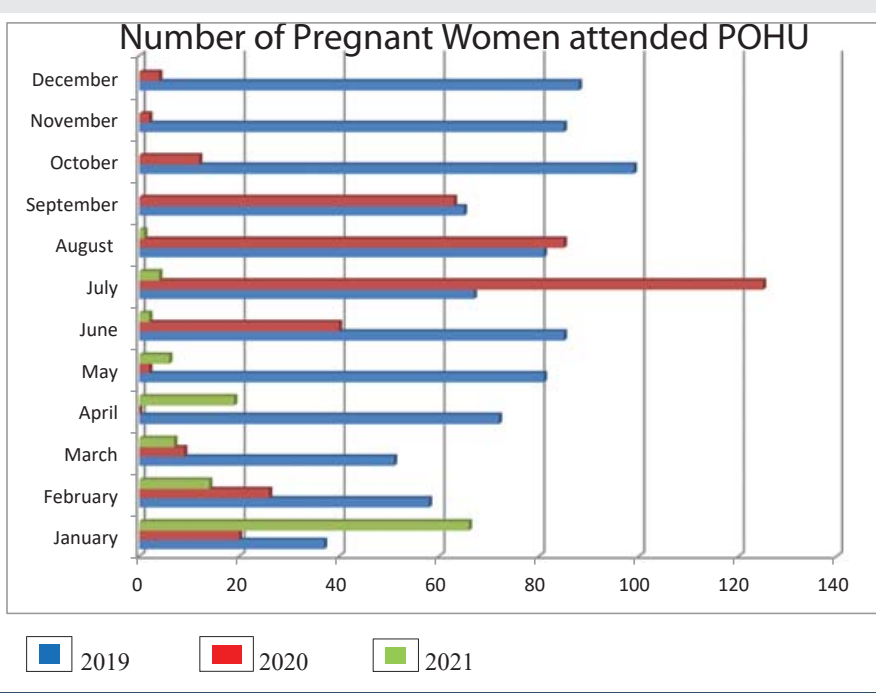

Figure 1: Attendance of pregnant women for preventive oral health care of $\mathrm{POHU}$ for 2019, 2020 and 2021 (up to August).

Citation: Perera I, Perera M, Ratnasekera N, Gajanayake C, Epa M (2021) Preventive oral health care for mitigating the CoVID-19 induced adverse maternal outcomes: View point from Sri Lanka on opportunities in crisis. J Gynecol Res Obstet 7(3): 036-041. DOI: https://dx.doi.org/10.17352/jgro.000103 
suggested by accumulating evidence on plausible mechanisms and epidemiological and molecular evidence on severity of Covid-19 and periodontitis, it could be well argued that poor oral health status and periodontal disease burden could elevate the risks of Covid-19 induced adverse maternal outcomes. On the other hand, the translational value of such evidence embrace the possibility of lowering the risks by improved oral health status and oral health promotion across pregnancy and throughout.

\section{Innovative strategies in providing preventive oral health care for pregnant women in Covid-19 era: Sri Lankan tertiary care public dental hospital experience}

Availability of and accessibility to preventive oral health care services become problematic for pregnant women in Covid-19 stricken scenarios. Furthermore, conducting community based oral health sessions at antenatal clinics needs deferment in the light of directives issued by health authorities on streamlining oral health care services for essential and emergency services. Moreover, the supportive staff predominantly the health assistants (dental surgery assistants) of the dental hospital has been deployed for patient care services of Covid -19 treatment centres since the inception of the pandemic. At the time of writing of this opinion, one health assistant out of two assistants working at POHU has been deployed for Covid-19 patient care services. Therefore, novel approaches in providing preventive oral health care for pregnant women have become the need of the hour. Few oral health sessions at selected CMC antenatal clinics were conducted since January to May 2021, with small groups of attendees and adhering to physical distancing measures. Moreover, the pregnant women whom were referred to POHU were advised to make telephone calls and book an appointment. The majority of referred women needed full mouth scaling routinely done by ultrasonic scaling machine, despite the high risk of aerosol generation of the procedure, treatment was provided after triaging and adeherence to infection control measures. All the pregnant women were provided with customized oral hygiene instructions and dietary advice in addition to fluoride gel application and filling for teeth with cavities. Those who needed tooth extractions and root canal treatment were referred to outpatient dental clinics and restorative consultant units. Moreover, hand scaling was performed for some pregnant women as there were frequent breakdown of a dental unit with the ultrasonic scaling machine.

An innovative approach of over- the- phone preventive oral health care package was delivered for randomly selected pregnant women whom were in various trimesters of their advancing pregnancies from the data base of POHU. This was customized to the oral health issues of a given woman mostly confined to homes in lock down scenarios. The recipients welcomed this service appraising it as beyond their expectations amidst the crisis of Covid-19. One recipient was nearing her date to deliver her baby at a leading private Women and Children's Hospital in Sri Lanka. She was struggling to reach the hotline of the given hospital to get the details of her real time PCR testing prior to be admitted for the delivery for the planned C-section.
Therefore, our approach helped this recipient to access necessary information by navigating available services. As evident from this experience, over-the- phone preventive oral care services provided a promising approach embraced with supportive care for discerning pregnant women in Covid-19. Nevertheless, further evaluations of such services warranted for evidencebased conclusions. Improving oral health of pregnant women underpinned by preventive oral health care package could be a way forward to reduce the severity of Covid-19 symptoms and associated morbidity supported by recent evidence [23]. This becomes applicable even for those who are vaccinated as vaccination may not totally prevent catching the infection in epidemic scenarios.

In conclusion, it could be rational to speculate that preventive oral health care services targeted to pregnant women could offer unexplored opportunities in Covid -19 crisis. In the light of accumulating research evidence on links between poor oral health status, periodontitis and Covid-19 complications and adverse outcomes, preventive oral health care has a promising potential to mitigate some of those. Nevertheless, further research warranted in this regard with methodological rigour for more conclusive evidence.

\section{References}

1. Wastnedge EAN, Reynolds RM, van Boeckel SR, Stock SJ, Denison FC, et al. (2021) Pregnancy and COVID-19. Physiol Rev 101: 303-318. Link: https://bit.ly/3IAzBKc

2. Alzamora MC, Paredes T, Caceres D, Webb CM, Valdez LM, et al. (2020) Severe COVID-19 during Pregnancy and Possible Vertical Transmission. Am J Perinatol 37: 861-865. Link: https://bit.ly/3BEzfb9

3. Juan J, Gil MM, Rong Z, Zhang Y, Yang H, et al. (2020) Effect of coronavirus disease 2019 (COVID-19) on maternal, perinatal and neonatal outcome: systematic review. Ultrasound Obstet Gynecol 56: 15-27. Link: https://bit.ly/3v9wGLS

4. Zaigham M, Andersson $O$ (2020) Maternal and perinatal outcomes with COVID-19: A systematic review of 108 pregnancies. Acta Obstet Gynecol Scand 99: 823-829. Link: https://bit.ly/3oZgSKf

5. Hessami K, Homayoon N, Hashemi A, Vafaei H, Kasraeian M, et al. (2020) COVID-19 and maternal, fetal and neonatal mortality: a systematic review. J Matern Fetal Neonatal Med 16: 1-6. Link: https://bit.ly/2YPKLIJ

6. Karunachandra NN, Perera IR, Fernando G (2012) Oral health status during pregnancy: rural-urban comparisons of oral disease burden among antenatal women in Sri Lanka. Rural Remote Health 12: 1902. Link: https://bit.ly/3FHnuTH

7. Africa CWJ, Turton M (2019) Oral Health Status and Treatment Needs of Pregnant Women Attending Antenatal Clinics in KwaZulu-Natal, South Africa. Int J Dent 2019: 5475973. Link: https://bit.ly/3AEPWln

8. Erchick DJ, Rai B, Agrawal NK, Khatry SK, Katz J, et al. (2019) Oral hygiene, prevalence of gingivitis, and associated risk factors among pregnant women in Sarlahi District, Nepal. BMC Oral Health 19: 2. Link: https://bit.ly/3oUfzMV

9. Abariga SA, Whitcomb BW (2016) Periodontitis and gestational diabetes mellitus: a systematic review and meta-analysis of observational studies. BMC Pregnancy Childbirth 16: 344. Link: https://bit.ly/3BEFnQu

10. Xiong X, Buekens P, Fraser WD, Beck J, Offenbacher S (2006) Periodontal disease and adverse pregnancy outcomes: a systematic review. BJOG 113: 135-143. Link: https://bit.ly/3v6MC1e 
11. Ide M, Papapanou PN (2013) Epidemiology of association between maternal periodontal disease and adverse pregnancy outcomes--systematic review. J Clin Periodontol 40: S181- S194. Link: https://bit.ly/3mSELRh

12. Jakovljevic A, Sljivancanin Jakovljevic T, Duncan HF, Nagendrababu V, et al. (2021) The association between apical periodontitis and adverse pregnancy outcomes: a systematic review. Int Endod J 54: 1527-1537. Link: https://bit.ly/3Dt4y9k

13. Gomez-Arango LF, Barrett HL, McIntyre HD, Callaway LK, Morrison M, et al (2017) Contributions of the maternal oral and gut microbiome to placental microbial colonization in overweight and obese pregnant women. Sci Rep 7 2860. Link: https://go.nature.com/3oXCXZL

14. Dessì A, Bosco A, Pintus R, Orrù G, Fanos V (2021) Fusobacterium nucleatum and alteration of the oral microbiome: from pregnancy to SARS-COV-2 infection. Eur Rev Med Pharmacol Sci 25: 4579-4596. Link: https://bit.ly/3DR2ZSX

15. Cobb CM, Kelly PJ, Williams KB, Babbar S, Angolkar M, et al. (2017) The oral microbiome and adverse pregnancy outcomes. Int $\mathrm{J}$ Womens Health 9: 551 559. Link: https://bit.ly/3FK5I20

16. Perera IR, Perera ML, Ratnasekera R (2018) The Pregnancy Microbiome: The Link Between Maternal Periodontitis and Adverse Pregnancy Outcomes: Opinion. Invest Gynecol Res Women's Health 2. Link: https://bit.ly/3IABAhC

17. Kardeh B, von Bergmann H, Brondani M (2021) Pregnant women's perspectives on integrating preventive oral health in prenatal care. BMC Pregnancy Childbirth 21: 271. Link: https://bit.ly/3v7V00u

18. Marouf N, Cai W, Said KN, Daas H, Diab H, et al. (2021) Association between periodontitis and severity of COVID-19 infection: A case-control study. J Clin Periodontol 48: 483-491. Link: https://bit.ly/3DCBRHa

19. Anand PS, Jadhav P, Kamath KP, Kumar SR, Vijayalaxmi S, Anil S (2021) A case-control study on the association between periodontitis and coronavirus disease (COVID-19). J Periodontol. Link: https://bit.ly/3IASRar

20. Barek MA, Aziz MA, Islam MS (2020) Impact of age, sex, comorbidities and clinical symptoms on the severity of COVID-19 cases: A meta-analysis with 55 studies and 10014 cases. Heliyon 6: e05684. Link: https://bit.ly/3BF9ffw

21. Sanz M, Marco Del Castillo A, Jepsen S, Gonzalez-Juanatey JR, et al (2020) Periodontitis and cardiovascular diseases: Consensus report. J Clin Periodontol 47: 268-288. Link: https://bit.ly/3FF0l4d

22. Kapila YL (2021) Oral health's inextricable connection to systemic health Special populations bring to bear multimodal relationships and factors connecting periodontal disease to systemic diseases and conditions. Periodontol 87: 11-16. Link: https://bit.ly/30dkFJG

23. Botros N, lyer P, Ojcius DM (2020) Is there an association between ora health and severity of COVID-19 complications? Biomed J 43: 325-327. Link: https://bit.ly/3iVEn38

24. Pitones-Rubio V, Chávez-Cortez EG, Hurtado-Camarena A, González-Rascón A, Serafín-Higuera N (2020) Is periodontal disease a risk factor for severe COVID-19 illness? Med Hypotheses 144: 109969. Link: https://bit.ly/3FK8nZN

25. Shamsoddin E (2021) Is periodontitis associated with the severity of COVID-19? Evid Based Dent 22: 66-68. Link: https://go.nature.com/3AC5S7S

26. Takahashi Y, Watanabe N, Kamio N, Yokoe S, Suzuki R, et al. (2021) Expression of the SARS-CoV-2 Receptor ACE2 and Proinflammatory Cytokines Induced by the Periodontopathic Bacterium Fusobacterium nucleatum in Human Respiratory Epithelial Cells. Int J Mol Sci 22: 1352. Link: https://bit.ly/3ACOh1s

27. Adeniyi AA, Laronde DM, Brondani M, Donnelly L (2020) Perspectives of socially disadvantaged women on oral healthcare during pregnancy. Community Dent Health 37: 39-44. Link: https://bit.ly/3FLvOC2

28. Perera IR, Perera ML, Kulathunge T (2019) Providing Oral Health Awareness and Screening to Low Income, Urban Pregnant Women: A Developing Country Perspective. JGWH 17: 555958. Link: https://bit.ly/3FK8VyP

29. Smith O (2018) "Sri Lanka: Achieving Pro-Poor Universal Health Coverage without Health Financing Reforms". Universal Health Coverage Study Series No. 38, World Bank Group, Washington, DC. Link: https://bit.ly/3v5lezs

30. Department of Census and Statistics Sri Lanka. Link: https://bit.ly/3AAm2i7

31. Family Health Bureau, Ministry of Health (2009) Oral health care during pregnancy. Practice Guidelines. Colombo, Sri Lanka: Family Health Bureau, Ministry of Health.

32. Ratnasekera N, Perera I, Kandapolaarachchige P, Surendra G, Dantanarayana A (2020) Supportive care for oral cancer survivors in COVID-19 lockdown. Psychooncology 29: 1409-1411. Link: https://bit.ly/2YRFIkP

33. Dantanarayana AK, Perera IR (2021) Profile of Surgical Oncological Patient Care Services Provided for Oral Cancer Patients by a Premier Public Dental Hospital in Sri Lanka Amidst COVID-19 Pandemic. Oral Health Dental Sci 5 1-4. Link: https://bit.ly/30ovjNV

34. Surendra G, Perera I, Ranasinghe A, Kumarapeli V, Tham R, Wickramaratne P (2021) Pattern and Causes of Oral and Maxillofacial Injuries Presented to a Tertiary Care Public Dental Hospital in Strictly Imposed COVID-19 Lockdown Scenario. Oral 1: 3-14. Link: https://bit.ly/3v5IJtk

35. Perera I, Ratnasekera N, Nanayakkara A, Surendra G, Gajanayake C (2020) Child Friendly Preventive Oral Health Care Package to facilitate COVID-19 Lock-Down Social Distancing Strategy: Evidence from Sri Lanka. Am J Den and Ora Car 3: 01-04. Link: https://bit.ly/3v8WszD

\section{Discover a bigger Impact and Visibility of your article publication with} Peertechz Publications

\section{Highlights}

* Signatory publisher of ORCID

* Signatory Publisher of DORA (San Francisco Declaration on Research Assessment)

- Articles archived in worlds' renowned service providers such as Portico, CNKI, AGRIS, TDNet, Base (Bielefeld University Library), CrossRef, Scilit, J-Gate etc.

* Journals indexed in ICMJE, SHERPA/ROMEO, Google Scholar etc.

* OAI-PMH (Open Archives Initiative Protocol for Metadata Harvesting)

* Dedicated Editorial Board for every journa

* Accurate and rapid peer-review process

* Increased citations of published articles through promotions

- Reduced timeline for article publication

Submit your articles and experience a new surge in publication services (https://www.peertechz.com/submission).

Peertechz journals wishes everlasting success in your every endeavours.

Citation: Perera I, Perera M, Ratnasekera N, Gajanayake C, Epa M (2021) Preventive oral health care for mitigating the COVID-19 induced adverse maternal outcomes: View point from Sri Lanka on opportunities in crisis. J Gynecol Res Obstet 7(3): 036-041. DOI: https://dx.doi.org/10.17352/jgro.000103 